\title{
Topological aspect of black hole with Skyrme hair
}

\author{
Yi-Shi Duan, Xin-Hui Zhang** and Li Zhao \\ Institute of Theoretical Physics, Lanzhou University \\ Lanzhou 730000, People's Republic of China
}

\begin{abstract}
Based on the $\phi$-mapping topological current theory, we show that the presence of the black hole leaves fractional baryon charge outside the horizon in the Einstein-Skyrme theory. A topological current is derived from the Einstein-Skyrme system, which corresponds to the monopoles around the black hole. The branch process (splitting, merging and intersection) is simply discussed during the evolution of the monopoles.
\end{abstract}

PACS numbers: 04.70.Bw, 11.27.+d, 12.39.Dc

keywords: Black hole; skyrmion; monopoles.

*Electronic address: zhangxingh03@st.lzu.edu.cn 


\section{INTRODUCTION}

The famous conjecture "Black holes have no hair" is that black holes are uniquely specified by the conserved quantities: mass, angular momentum, and charge [1]. Investigating these "no-hair" theorems, however, shows that while powerful, they are not omnipotent. It was pointed out by Bowick et. al. [2] that there exists a family of Schwarzschild black-hole solutions to the Einstein-axion equations labelled by a conserved topological charge. Such black holes could be said to be carrying axion hair. It was then rapidly realized that the same fractional charge that could give rise to enhancement of proton decay catalysis [3, 44, 5]. The full ramifications of this type of quantum hair have been most eloquently argued by Coleman et. al. [6, 7].

The Skyrme model is an effective meson theory [8, 9] where the baryons are identified with topological solitons, so-called skyrmions. The baryon number $B$ corresponds to the topological charge [10]. Thus the model gives a unified description of hadron physics in terms of meson fields [11]. The advantage of the Skyrme model to more realistic nucleon models is that it is straightforward to couple to gravity, that is, the Einstein-Skyrme model [12]. The Einstein-Skyrme model was firstly studied by Luckock and Moss [13] where the Schwarzschile black hole with Skyrme hair was obtained. This is another counter example of the no-hair conjecture for black holes [1]. The full Einstein-Skyrme system was solved later to obtain spherically symmetric black holes with Skyrme hair [14] and regular gravitating skyrmions [15].

Here we shall be particular interested in the Einstein-Skyrme model and the hair of the black hole. We reexamine some work in detail using the $\phi$-mapping topological current theory and present there leaves fractional baryon charges outside the horizon of black hole in the Einstein-Skyrme model. Meanwhile, in this system there also exist monopoles, whose quantum number is determined by Hopf indices and Brouwer degrees. Thus, in some sense, such black holes could be said to be carrying Skyrme hair and monopole hair at the same time.

This paper is arranged as follows. In Sec. III, we research the topological current in the Einstein-Skyrme model and reveal that it can be decomposed into two parts. One is the usual baryon number current, i.e., the skyrmion current. The other is the monopole current. In Sec. III, We study the baryon number current and point out that baryon number may be 
fractional outside the horizon. In Sec. IV, using the $\phi$-mapping topological current theory, we investigate the inner topological structure of the monopole current and simply discuss the branch process during the evolution of the monopoles. The conclusion of this paper is given in Sec. V.

\section{TOPOLOGICAL CURRENT IN EINSTEIN-SKYRME MODEL}

We write the field $U$ in $\mathrm{SU}(2)$ space in a general form

$$
\begin{aligned}
& U=e^{i n F(\varphi)}=\cos F+i n \sin F, \\
& n=n^{a} \sigma_{a}, \quad n^{a}=\frac{\phi^{a}}{\|\phi\|},
\end{aligned}
$$

where $\phi^{a}(a=1,2,3)$ are three fundamental functions with space-time coordinates $x^{\mu}$ and $\sigma^{a}$ are pauli matrices. Then the Einstein-Skyrme model is denoted by the Lagrangian

$$
\begin{aligned}
L & =L_{G}+L_{S} \\
& =\frac{R}{16 \pi G}+\frac{F_{\pi}}{16} g^{\mu \nu} \operatorname{tr}\left(L_{\mu} L_{\nu}\right)+\frac{1}{32 a^{2}} g^{\mu \nu} g^{\rho \sigma} \operatorname{tr}\left(\left[L_{\mu}, L_{\rho}\right]\left[L_{\nu}, L_{\sigma}\right]\right),
\end{aligned}
$$

in which $R$ is the scalar curvature, $G$ is the Newton's constant and $L_{\mu}=U^{+} \partial_{\mu} U$. It is well known that the Einstein-Skyrme model has black hole solutions [13, 14, 15]. Here we leave out the concrete process of solving the Einstein equations and directly explore the topological properties of black hole solutions in the Einstein-Skyrme system. Correspondingly, we shall impose the spherically symmetric ansatz on the metric

$$
d s^{2}=-N^{2}(r) C(r) d t^{2}+\frac{1}{C(r)} d r^{2}+r^{2} d \Omega^{2},
$$

where $C(r)$ is defined as follows:

$$
C(r)=1-\frac{2 G m(r)}{r}
$$

At the horizon $r=r_{h}$, we have $C\left(r_{h}\right)=0$, that is, $m\left(r_{h}\right)=r_{h} / 2 G$. It should be noted that in the presence of black hole, the matter field is defined only outside the horizon and therefore the integral over the space is performed from the horizon to infinity. The covariant topological current in this model is defined by [8, 9]

$$
B^{\mu}=\frac{\epsilon^{\mu \nu \lambda \rho}}{24 \pi^{2}} \frac{1}{\sqrt{-g}} \operatorname{tr}\left[U^{-1}\left(\partial_{\nu} U\right) U^{-1}\left(\partial_{\lambda} U\right) U^{-1} \partial_{\rho} U\right]
$$


whose 0-th component can be expressed as

$$
B(U)=\frac{\epsilon^{0 i j k}}{24 \pi^{2}} \frac{1}{\sqrt{-g}} \operatorname{tr}\left[U^{-1}\left(\partial_{i} U\right) U^{-1}\left(\partial_{j} U\right) U^{-1} \partial_{k} U\right], \quad i, j, k=1,2,3,
$$

which is just the topological current density. $B(U)$ defined by Eq. (7) is additive

$$
B\left(U_{1} U_{2}\right)=B\left(U_{1}\right)+B\left(U_{2}\right)
$$

Substituting Eq. (2) into Eq. (6) and taking notice of the relations: $n^{2}=1$ and $n \partial_{\mu} n+$ $\partial_{\mu} n n=0$, we find $B^{\mu}$ is composed of two parts

$$
B^{\mu}=B_{1}^{\mu}+B_{2}^{\mu}
$$

where

$$
B_{1}^{\mu}=\frac{1}{4 \pi^{2}} \frac{1}{\sqrt{-g}} \epsilon^{\mu \nu \lambda \rho} \epsilon_{a b c} \sin ^{2} F \partial_{\nu} F n^{a} \partial_{\lambda} n^{b} \partial_{\rho} n^{c}
$$

and

$$
B_{2}^{\mu}=\frac{1}{12 \pi^{2}} \frac{1}{\sqrt{-g}} \epsilon^{\mu \nu \lambda \rho} \epsilon_{a b c} \sin ^{3} F \cos F \partial_{\nu} n^{a} \partial_{\lambda} n^{b} \partial_{\rho} n^{c} .
$$

We shall see later that the current $B_{1}^{\mu}$ denoted by Eq. (10) is identical with the baryon number current, i.e. the skyrmion current [16], while the current $B_{2}^{\mu}$ denoted by Eq. (11) corresponds to the monopoles with charge $g=(2 / 3 \pi) \sin ^{3} F \cos F$.

\section{FRACTIONAL BARYON CHARGES}

In this section, we discuss the baryon number current $B_{1}^{\mu}$. From Eq. (10) we find the baryon number density

$$
\rho(x)=\frac{1}{\sqrt{-g}} \rho(\phi) D\left(\frac{\phi}{x}\right)
$$

where

$$
\rho(\phi)=\frac{1}{2 \pi^{2}} \frac{\sin ^{2} F}{\phi^{2}} \frac{d F}{d \phi}, \quad D\left(\frac{\phi}{x}\right)=\frac{1}{2} \epsilon^{i j k} \epsilon_{a b c} \partial_{i} n^{a} \partial_{j} n^{b} \partial_{k} n^{c}
$$

Then the baryon number can be expressed as

$$
B_{1}=\int_{V} \rho(x) \sqrt{-g} d^{3} x=\int_{T} \rho(\phi) d^{3} \phi
$$

The above integral shows that the fundamental field $\phi$ maps $V$ into $T$ in iso-space. We

consider the case that while the vector $\vec{x}$ covers the region $V$ once, function $\phi$ covers the 
corresponding elementary region $T_{0} N$ times (i.e. $T=N T_{0}$ ) and impose the boundary conditions on the profile function

$$
F(\infty)=0, \quad F\left(r_{h}\right)=F_{h},
$$

which determines the value at the horizon $r_{h}$. Then from Eq. (12) and Eq. (14), we obtain the baryon number

$$
B_{1}=\frac{2 N}{\pi} \int_{0}^{F_{h}} \sin ^{2} F d F=\frac{N}{2 \pi}\left(2 F_{h}-\sin 2 F_{h}\right) .
$$

If $F_{h}=\pi$, the baryon number is still an integer. This configuration represents a proton tightly bound to the black hole. On the other hand, if $F_{h}<\pi$, the baryon number may not be an integer and the skyrmion can carry fractional baryon number. This configuration will be interpreted as a proton partially swallowed by the black hole.

\section{MONOPOLES OUTSIDE THE HORIZON}

\section{A. The generation of the monopole}

Generally speaking, one explores the topological feature of the Einstein-Skyrme model only by the baryon current $B_{1}^{\mu}$. In Sec. III, we obtain another current $B_{2}^{\mu}$, i.e. monopole topological current, which contributes rich properties to the black hole with skyrmion hair. In this subsection, using the $\phi$-mapping topological current theory, we investigate the inner topological structure of $B_{2}^{\mu}$. Eq. (11) can be rewritten as

$$
B_{2}^{\mu}=\frac{g_{0}}{8 \pi} \frac{1}{\sqrt{-g}} \epsilon^{\mu \nu \lambda \rho} \epsilon_{a b c} \partial_{\nu} n^{a} \partial_{\lambda} n^{b} \partial_{\rho} n^{c}
$$

where $g_{0}=(2 / 3 \pi) \sin ^{3} F \cos F$. Taking account of Eq. (2) and using $\partial_{\mu} n^{a}=\phi^{a} \partial_{\mu}(1 /\|\phi\|)+$ $(1 /\|\phi\|) \partial_{\mu} \phi^{a}$, Eq. (17) can be expressed as

$$
B_{2}^{\mu}=\frac{g_{0}}{8 \pi} \frac{1}{\sqrt{-g}} \epsilon^{\mu \nu \lambda \rho} \epsilon_{a b c} \frac{\partial}{\partial \phi^{l}} \frac{\partial}{\partial \phi^{a}}\left(\frac{1}{\|\phi\|}\right) \partial_{\nu} \phi^{l} \partial_{\lambda} \phi^{b} \partial_{\rho} \phi^{c} .
$$

If we define Jacobian $\epsilon^{l b c} D^{\mu}\left(\frac{\phi}{x}\right)=\epsilon^{\mu \nu \lambda \rho} \partial_{\nu} \phi^{l} \partial_{\lambda} \phi^{b} \partial_{\rho} \phi^{c}$, and make use of the Green function relation in $\phi$ space: $\frac{\partial}{\partial \phi^{a}} \frac{\partial}{\partial \phi^{a}}\left(\frac{1}{\|\phi\|}\right)=-4 \pi \delta(\vec{\phi})$, we obtain the $\delta$-function like $B_{2}^{\mu}[17,18,19]$

$$
B_{2}^{\mu}=\frac{1}{\sqrt{-g}} g_{0} \delta(\vec{\phi}) D^{\mu}\left(\frac{\phi}{x}\right) .
$$


The expression of Eq. (19) provides an important conclusion:

$$
B_{2}^{\mu}\left\{\begin{array}{l}
=0, \text { if and only if } \vec{\phi} \neq 0 . \\
\neq 0, \text { if and only if } \vec{\phi}=0 .
\end{array}\right.
$$

So it is necessary to study the zero points of $\vec{\phi}$ to determine the nonzero solutions of $B_{2}^{\mu}$. The implicit function theory [20] shows that under the regular condition

$$
D^{\mu}(\phi / x) \neq 0
$$

the general solutions of

$$
\phi^{1}\left(t, x^{1}, x^{2}, x^{3}\right)=0, \quad \phi^{2}\left(t, x^{1}, x^{2}, x^{3}\right)=0, \quad \phi^{3}\left(t, x^{1}, x^{2}, x^{3}\right)=0
$$

can be expressed as

$$
x^{1}=x_{k}^{1}(t), \quad x^{2}=x_{k}^{2}(t), \quad x^{3}=x_{k}^{3}(t),
$$

which represent the world lines of $N$ moving isolated singular points $\vec{x}_{k}(k=1,2,3 \cdots N)$. These singular solutions are just the monopoles located at the zero point of field $\vec{\phi}$ outside the horizon of the black holes. Then questions are raised naturally: what are the topological charges of the monopoles and how to obtain the inner structure of $B_{2}^{\mu}$. Now, we investigate the topological charges of the monopoles and their quantization. Let $M_{k}$ be a neighborhood of $\vec{x}_{k}$ with boundary $\partial M_{k}$ satisfying $\vec{x}_{k} \nsubseteq \partial M_{k}, M_{i} \cap M_{k}=\varnothing$. Then the generalized winding number $W_{k}$ of $n^{a}(\vec{x})$ at $\vec{x}_{k}$ can be defined by the Gauss map [21] $n: \partial M_{k} \rightarrow S^{2}$,

$$
W_{k}=\frac{1}{8 \pi} \int_{\partial M_{k}} n^{*}\left(\epsilon_{a b c} n^{a} d n^{b} \wedge d n^{c}\right),
$$

where $n^{*}$ is the pull back of map $n$. The generalized winding number is a topological invariant and is also called the degree of Gauss map. It is well known that $W_{k}$ are corresponding to the second homotopy group $\pi_{2}\left(S^{2}\right)=Z$ ( the set of integers). Using the Stokes' theorem in exterior differential form and the result in Eq. (19), we get

$$
W_{k}=\int_{M_{k}} \delta(\vec{\phi}) D\left(\frac{\phi}{x}\right) d^{3} x .
$$

In order to explore the inner topological structure of $B_{2}^{\mu}$, one can expand the $\delta(\vec{\phi})$ as:

$$
\delta(\vec{\phi})=\sum_{k=1}^{N} c_{k} \delta\left(\vec{x}-\vec{x}_{k}\right),
$$


where the coefficients $c_{k}$ must be positive, i.e., $c_{k}=\left|c_{k}\right|$. Substituting Eq. (26) into Eq. (25) and calculating the integral, we obtain an expression for $c_{k}$,

$$
c_{k}=\frac{\left|W_{k}\right|}{|D(\phi / x)|_{\vec{x}=\vec{x}_{k}}} .
$$

Then $\delta(\vec{\phi})$ can be reexpressed as

$$
\delta(\vec{\phi})=\sum_{k=1}^{N} \frac{\beta_{k}}{\left|D\left(\frac{\phi}{x}\right)\right|_{\vec{x}=\vec{x}_{k}}} \delta\left(\vec{x}-\vec{x}_{k}\right)
$$

in which the positive integer $\beta_{k}=\left|W_{k}\right|$ is the Hopf index of $\phi$-mapping, which means that when $\vec{x}$ covers the neighborhood of the zero point $\vec{x}_{k}$ once, the vector field $\vec{\phi}$ covers the corresponding region in $\phi$ space $\beta_{k}$ times. It can be proved from Eq. (23) that the velocity of the $k$ th zero is determined by [22]

$$
V^{\mu}=\frac{d x_{k}^{\mu}}{d t}=\left.\frac{D^{\mu}\left(\frac{\phi}{x}\right)}{D^{0}\left(\frac{\phi}{x}\right)}\right|_{\vec{x}=\vec{x}_{k}}
$$

where $D^{0}\left(\frac{\phi}{x}\right)$ is the usual Jacobian $D\left(\frac{\phi}{x}\right)$. Then substituting Eqs. (29) and (28) into Eq. (19), we get the dynamic form of the current $B_{2}^{\mu}$

$$
B_{2}^{\mu}=\frac{1}{\sqrt{-g}} g_{0} \sum_{k=1}^{N} \beta_{k} \eta_{k} \delta\left(\vec{x}-\vec{x}_{k}(t)\right) \frac{d x_{k}^{\mu}}{d t}=\rho V^{\mu}
$$

and the topological charge

$$
B_{2}=\int_{V} \rho \sqrt{-g} d^{3} x=\int_{V} g_{0} \sum_{k=1}^{N} \beta_{k} \eta_{k} \delta\left(\vec{x}-\vec{x}_{k}(t)\right) d^{3} x=g_{0} \sum_{k=1}^{N} W_{k},
$$

where the integral over the space is from the horizon to infinity, $W_{k}=\beta_{k} \eta_{k}$ is the winding number and $\eta_{k}=\left.\operatorname{sgn} D(\phi / x)\right|_{\vec{x}_{k}}= \pm 1$ is called the Brouwer degree of the $\phi$-mapping at $\vec{x}_{k}$. From the above discussions, we see that:

(i) The monopoles are generated from the zeros of the field $\vec{\phi}$ outside the horizon of the black holes.

(ii) The monopoles are topologically quantized in the unit of the basic charge $g_{0}$ and the topological quantum numbers are determined by Hopf indices $\beta_{k}$ and Brouwer degrees $\eta_{k}$ of the $\phi$-mapping.

(iii) The Brouwer degree $\eta_{k}=+1$ corresponds to the monopoles, while $\eta_{k}=-1$ corresponds to the anti-monopoles. 


\section{B. The evolution of the monopoles}

In Sec. IVA, we have used the regular condition $D^{\mu}(\phi / x) \neq 0$. When this condition fails, branch process will occur. Here we discuss branch process during the evolution of monopoles. It is known that the velocity of the monopoles is given by

$$
V^{\mu}=\frac{d x^{\mu}}{d t}=\frac{D^{\mu}(\phi / x)}{D^{0}(\phi / x)}
$$

where

$$
\begin{aligned}
& D^{0}(\phi / x)=\epsilon_{a b c} \partial_{1} \phi^{a} \partial_{2} \phi^{b} \partial_{3} \phi^{c}, \quad D^{1}(\phi / x)=\epsilon_{a b c} \partial_{2} \phi^{a} \partial_{3} \phi^{b} \partial_{0} \phi^{c}, \\
& D^{2}(\phi / x)=\epsilon_{a b c} \partial_{3} \phi^{a} \partial_{0} \phi^{b} \partial_{1} \phi^{c}, \quad D^{3}(\phi / x)=\epsilon_{a b c} \partial_{0} \phi^{a} \partial_{1} \phi^{b} \partial_{2} \phi^{c} .
\end{aligned}
$$

From Eq. (32), it is obvious that when

$$
D^{0}(\phi / x)=0
$$

at the very point $\left(t^{*}, \vec{x}^{*}\right)$, the velocity

$$
\frac{d x^{1}}{d t}=\left.\frac{D^{1}(\phi / x)}{D^{0}(\phi / x)}\right|_{\left(t^{*}, \vec{x}^{*}\right)}, \quad \frac{d x^{2}}{d t}=\left.\frac{D^{2}(\phi / x)}{D^{0}(\phi / x)}\right|_{\left(t^{*}, \vec{x}^{*}\right)}, \frac{d x^{3}}{d t}=\left.\frac{D^{3}(\phi / x)}{D^{0}(\phi / x)}\right|_{\left(t^{*}, \vec{x}^{*}\right)}
$$

are not unique in the neighborhood of $\left(t^{*}, \vec{x}^{*}\right)$. This very point $\left(t^{*}, \vec{x}^{*}\right)$ is called the bifurcation point. Without loss of generality, we discuss only the branch of the velocity component $\left(d x^{1} / d t\right)$ at $\left(t^{*}, \vec{x}^{*}\right)$. It is known that the Taylor expansion of the solution of Eq. (23) in the neighborhood of $\left(t^{*}, \vec{x}^{*}\right)$ can generally be expressed as

$$
A\left(x^{1}-x^{1 *}\right)^{2}+2 B\left(x^{1}-x^{1 *}\right)\left(t-t^{*}\right)+C\left(t-t^{*}\right)^{2}+\cdots=0,
$$

where $A, B$ and $C$ are three constants. Then the above Taylor expansion leads to

$$
A\left(\frac{d x^{1}}{d t}\right)^{2}+2 B \frac{d x^{1}}{d t}+C=0 \quad(A \neq 0) .
$$

The solutions of Eq. (36) give different motion directions of the zero point at the bifurcation point. There are two possible cases:

(i) For $\Delta=4\left(B^{2}-A C\right)=0$, from Eq. (36) we get only one motion direction of the zero point at the bifurcation point: $\left.\left(d x^{1} / d t\right)\right|_{1,2}=-B / A$, which includes three sub-cases:

(a) One point defect splits into two points; 
(b) Two points merge into one;

(c) Two point defects tangentially intersect at the bifurcation point.

(ii) For $\Delta=4\left(B^{2}-A C\right)>0$, from Eq. (36) we get two different motion directions of the zero point: $\left.\left(d x^{1} / d t\right)\right|_{1,2}=\left(-B \pm \sqrt{B^{2}-A C}\right) / A$. This is the intersection of two point defects, which means that the two defects meet and then depart at the bifurcation point.

In both cases (i) and (ii), we know that the sum of the topological charges of final defects must be equal to that of the initial defects at the bifurcation point.

\section{CONCLUSION}

In this paper, we investigate the topological aspect of black hole with Skyrme hair in Einstein-Skyrme model. It is revealed that the baryon number may be fractional outside the horizon and there exist monopoles, whose topological charges are topologically quantized by Hopf indices and Brower degrees. Starting with the Einstein-Skyrme model, which supports black hole solutions, we discuss the baryon number current $B_{1}^{\mu}$ outside the horizon of the black hole and come to the conclusion: if the baryon number is still an integer, this configuration represents a proton tightly bound to the black hole; On the other hand, if the baryon number is not an integer, i.e. the skyrmion carries fractional baryon number, this configuration will be interpreted as a proton partially swallowed by the black hole. Meanwhile we find there is a topological current $B_{2}^{\mu}$ accompanied by the baryon number current $B_{1}^{\mu}$. We research the current $B_{2}^{\mu}$ in detail and point out that it corresponds to monopoles outside the horizon. By introducing the $\phi$-mapping topological current theory, we see that the zeros of the field $\vec{\phi}$ are just the sources of the monopoles, and the monopoles are topologically quantized in the unit of the basic charge $g_{0}$ and the topological quantum numbers are determined by the Hopf indices $\beta_{k}$ and Brower degrees $\eta_{k}$. Finally, we simply discuss the branch process (splitting, merging and intersection) when the condition $D^{\mu}(\phi / x) \neq 0$ fails. The topological charges are preserved in the branch process during the evolution of the monopoles. 


\section{ACKNOWLEDGMENT}

It is a great pleasure to thank Doctor T. Y. Si and Doctor Y. X. Liu for numerous fruitful discussions. This work was supported by the National Natural Science Foundation of China and the Doctoral Foundation of the People's Republic of China.

[1] R. Ruffini and J. A. Wheeler, Physics Today 24, 30 (1971).

[2] M. Bowick, S. Giddings, J. Harvey, G. Horowitz and A. Strominger, Phys. Rev. Lett. 61, 2823 (1988).

[3] Ph. de Sousa Gerbert, Phys. Rev. D 40, 1346 (1989).

[4] M. Alford, J. March-Russel and F. Wilczek, Nucl. Phys. B 328, 140 (1989).

[5] L. Krauss and F. Wilczek, Phys. Rev. Lett. 62, 1221 (1989).

[6] S. Coleman, J. Preskill and F. Wilczek, Mod. Phys. Lett. A 6, 1631 (1991).

[7] S. Coleman, J. Preskill and F. Wilczek, Phys. Rev. Lett. 67, 1975 (1991).

[8] T. H. R. Skyrme, Proc. Roy. Soc. A 260, 127 (1961).

[9] T. H. R. Skyrme, Nucl. Phys. 31, 556 (1962).

[10] E. Witten, Nucl. Phys. B 223, 422 (1983).

[11] N. Shiiki and N. Sawado, Phys. Rev. D 71, 104031 (2005).

[12] N. Shiiki and N. Sawado, Class.Quant.Grav. 22, 3561 (2005).

[13] H. Luckock and I. Moss, Phys. Lett. B 176, 341 (1986).

[14] S. Drozn, M. Heusler and N. Stranmann, Phys. Lett. B 268, 371 (1991).

[15] P. Bizon and T. Chmaj, Phys. Lett. B 297, 55 (1992).

[16] F. Wilczek and A. Zee, Phys. Rev. Lett. 51, 2250 (1983).

[17] Y. S. Duan, H. Zhang and S. Li, Phys. Rev. B 58, 125 (1998).

[18] Y. S. Duan and H. Zhang, Eur. Phys. J. D 5, 47 (1999).

[19] Y. S. Duan, X. Liu and P. M. Zhang, J. Phys. A 36, 563 (2003).

[20] É. Goursat, A Course in Mathematical Analysis, translated by E. R. Hedrick, Vol. I. (Dover, New York, 1904).

[21] Y. S. Duan, Y. Jiang and T. Xu, Phys. Lett. A 252, 307 (1999).

[22] L. B. Fu, Y. S. Duan and H. Zhang, Phys. Rev. D 61, 045004 (2000). 